\title{
Evaluation of a promising new botanical adult mosquito repellent on horses
}

\author{
Talbalaghi $\mathrm{A}^{1,2 *}$, Ali $\mathrm{A}^{3}$ and Hassandoust $\mathrm{S}^{4}$ \\ ${ }^{1}$ Freelance Entomologist, Mosquito Control Operations - Citta di Alessandria, Alessandria, Italy \\ ${ }^{2}$ Former President, European Mosquito Control Association, Italy \\ ${ }^{3}$ Emeritus Professor, University of Florida, Mid-Florida Research and Education Center, Apopka, Florida, USA \\ ${ }^{4}$ Public Health Officer of Iran University of Medical Science, Shahriar Health Network, Iran
}

\begin{abstract}
Repel Care ${ }^{\circledR}$, a botanical adult mosquito repellent, containing primarily turmeric and eucalyptus oils, was topically applied at 3.4 and 6.9 grams $/ \mathrm{m}^{2}$ surface area on sports horses and evaluated to repel adult mosquitoes (predominantly Ochlerotatus caspius) in a horse stable situated amid a rice field area in Alessandria, south Piedmont, Italy. The repellent gave satisfactory results with an overall mean repellence of $62.3 \%$ of adult mosquitoes (range 29.1 to $88.4 \%$ ) during the four evenings of evaluation. Higher rates of application of the repellent may yield better results in terms of magnitude and persistence.
\end{abstract}

\section{Introduction}

In Piedmont, northern Italy, vast areas of wetlands exist during most part of the year as a result of rice cultivation (Figure 1). It is estimated that nearly 100,000 ha of irrigated rice fields prevail annually in the Province of Alessandria, Italy [1]); Italian Ministry of Agriculture 2005 [2]. These rice fields provide ideal habitats for the breeding of several mosquito species each year; specifically, several generations of Ochlerotatus caspius. This mosquito species is a ferocious biter and is capable of dispersing over vast areas from the breeding source; wind carried Oc. caspius can migrate up to $30 \mathrm{~km}$ or more from the breeding source [3].

Besides humans, numerous animals including horses with relatively large bodies suffer from mosquito bites and mosquito-related transmission of diseases. In many situations in Alessandria, the horse stables are nestled in areas heavily infested with mosquitoes; these

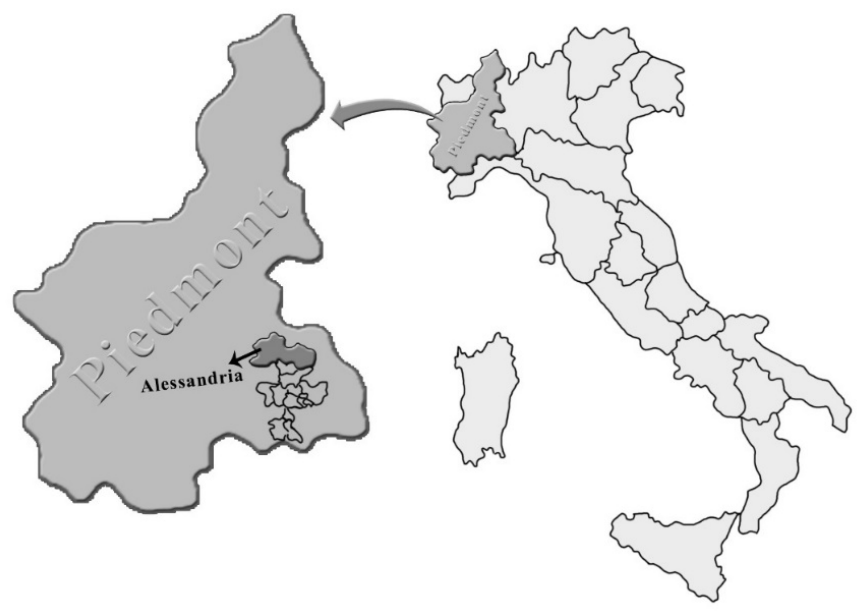

Figure 1. Map of Italy showing location of Piedmont and Alessandria stables are both open door and covered where the adult mosquitoes have free access. At present, mosquito control in and around the stables is mainly based on the use of chemical mosquito adulticides and synthetic repellents (information gathered from owners of stables and operators of private pest control companies in the area). These control measures have often resulted in unsatisfactory control of mosquitoes; besides, they are environmentally unacceptable due to their adverse effects. Alternately, natural botanical repellents provide ideal alternatives to these measures as these products have minimal adverse impact on the environment and the host animal [4].

In the present study, activity of a botanical adult mosquito repellent, Repel Care, against pedigree horses (expensive thoroughbred breed) in a stable in Alessandria, south Piedmont, was evaluated. In this area, the owners of the horses are usually nervous and greatly concerned about the discomfort and restlessness of their horses due to overwhelming mosquito bites as mosquito adulticiding and continued use of synthetic chemical repellents have failed to provide satisfactory results.

\section{Materials and Methods}

The test repellent, Repel Care ${ }^{\oplus}$, is a product of Thailand (containing accurately weighed raw materials according to batch formula). To make the formulation, known amounts of turmeric oil and eucalyptus oil are transferred into a mixer tank and blended until the formation of a homogeneous solution. Then PEG 400, ethanol and flavor are added to the mixture and the product is transferred to a packing room. The repellent essentially contained 38\% natural active ingredients (turmeric and eucalyptus oils) and had a pleasant odor.

${ }^{*}$ Correspondence to: Asghar Talbalaghi, Freelance Entomologist, Mosquito Control Operations - Citta di Alessandria, Alessandria, Italy, E-mail: talbalaghi@libero.it

Received: May 21, 2018; Accepted: May 25, 2018; Published: May 27, 2018 
Four horses (Figure 2) of the same age and body size (each horse head, neck, and total body surface area approximately $3 \mathrm{~m}^{2}$ ) were selected for this evaluation. These horses were generally of the same brownish color and were sports (race) horses (from CB Quarter Horse Training of Alessandria, Italy: CLASSICAL JUSTICE, CODY BENITO BAR, HOT AS I WANNA BE and LIL SMART PEPPY).

The evaluation was conducted for four consecutive evenings between 2000 and 2400 hours (five hours duration) for the first evening and thereafter between 20:00 and 01:00 hours (six hours duration) during summer 2005. Each evening, the repellent was applied topically on the entire head, neck and body of three horses, while the fourth horse was not treated with the repellent and served as a control. For an even coverage of the repellent on an entire treatment horse, the required amount of the repellent $\left(6.9 \mathrm{grams} / \mathrm{m}^{2}\right)$ was mixed with known amount of absolute alcohol and applied evenly on the horse skin. During the first treatment, a spray bottle was used to apply the repellent but due to the unexpected movements and disturbance by the horse, the distribution of the repellent on the horse was suspected uneven; therefore, the latter three treatments were gradually applied with a piece of cloth making sure of complete coverage and distribution of the repellent on each treatment horse. The control horse received absolute alcohol treatment only. The treatment rate of 6.9 grams $/ \mathrm{m}^{2}$ was applied in consideration of the recommended topical application rate of repellents on human skin [5] was evaluated during the first three evenings and on the fourth evening a rate of $3.4 \mathrm{grams} / \mathrm{m}^{2}$ was evaluated. The four horses were maintained tied under a shed in fixed positions during each evaluation period and any two horses were nearly $12 \mathrm{~m}$ apart from one another.

To monitor the number of mosquitoes landing and biting on each horse after the treatment, one technician was assigned to each horse for counting mosquitoes for a total of 10 minutes ( 5 minutes on each side of the horse) each hour during the total five or six hours of observation. During this observation, the number of mosquitoes on face, neck, different parts of the body, and legs of each horse were also noted. Every evening the location of horses was rotated (Figure 2) so that each horse occupied all locations at least once.

Adult mosquito repellence was calculated by using the following formula of Mulla suggested by Reison 2004, [6]

$\mathrm{R}=[(\mathrm{C}-\mathrm{T}) / \mathrm{C}] 100$. Where "C" is the total number of mosquitoes biting the control horse; and " $\mathrm{T}$ " the total number of mosquitoes biting the repellent-treated horse.

In order to assess the prevalence of adult mosquitoes in the vicinity of the experimental area, two $\mathrm{CO}_{2}$-baited traps were placed 50 meters away from the experimental area (Figure 3 ) and monitored hourly basis during the period of repellent evaluation for the prevailing (captured) mosquito populations in the area.

\section{Results}

Adult mosquito repellence from horses during the first three evenings of the repellent evaluation at $6.9 \mathrm{grams} / \mathrm{m}^{2}$ ranged from a mean value of $29.1 \%$ (first evening) to $88.4 \%$ (second evening), with an overall mean repellence of $62.3 \%$ during the first three evenings of evaluation. In the fourth evening, at $3.4 \mathrm{grams} / \mathrm{m}^{2}$, the mean repellence amounted to 59\% (Figures 4 and 5). Generally, the repellent activity was the highest during the second and third evenings of evaluation. During each evening, the number of mosquitoes in the controls was consistently higher at least during the first four hours of evaluation on each evening.

The data concerning $\mathrm{CO}_{2}$ traps varied widely during the different evenings but generally the number of adult mosquitoes captured during

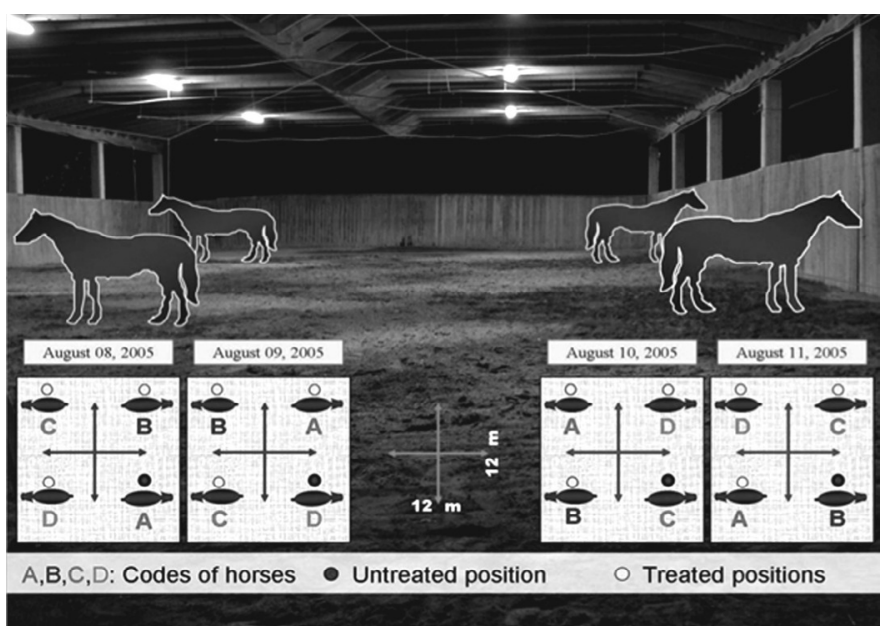

Figure 2. Repellent test horses ( $50 \mathrm{~m}$ apart from one another) in the test shed, Alessandria, Italy

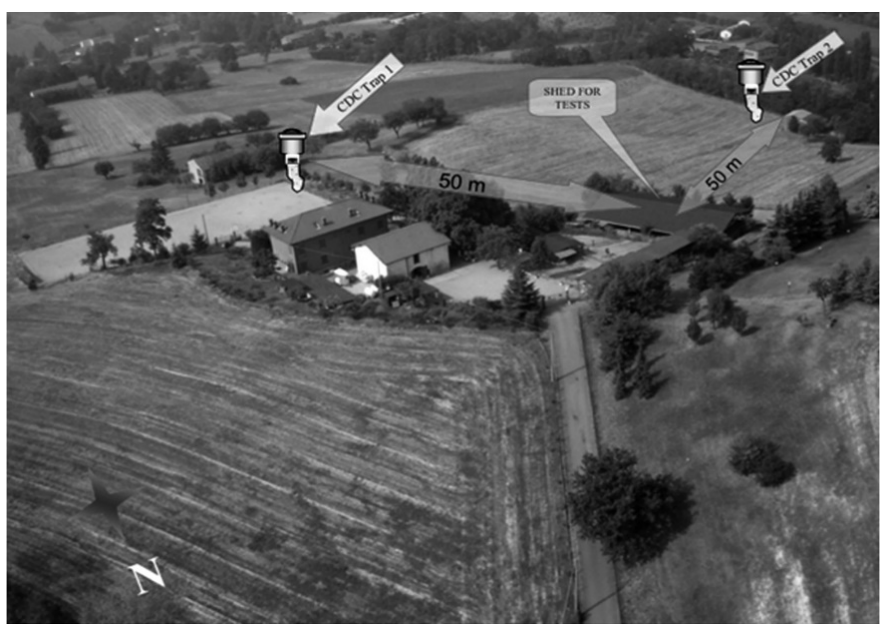

Figure 3. Placement of CDC traps $50 \mathrm{~m}$ away in north and east directions of the repellent test shed., Alessandria, Italy

the second and third hours of evaluation on any occasion was greater than the other hours of observation (Figures 6 and 7).

\section{Discussion}

"The use of repellent products to prevent insect and arthropod bites is probably proportional to the public perception of the threat from biting arthropods, whether the bthreat is from annoyance or risk of disease" [7]. Xue ET AL. 2007 [8] have provided detailed information on commercially available insect repellents and their use.

In this study, the entire horse body surface area of each experimental horse exposed to air was calculated. The quality of essential oils products available in the market and, specifically alcoholor water-based formulations may impair performance of the product, such as yield; chemical constituents and physical properties depend on many factors that affect the quality of essential oils, plant preparations and methods of extraction [9]. The hairy body of horses may be a helpful factor in retaining a repellent and its effects for a longer time period; however, the method of application of a repellent on the horse body may have its limitation, especially if applied by an inexperienced horse handler, Based on the results of the present study, it is suggested that the essential oils mixture used in the present work is valuable and 


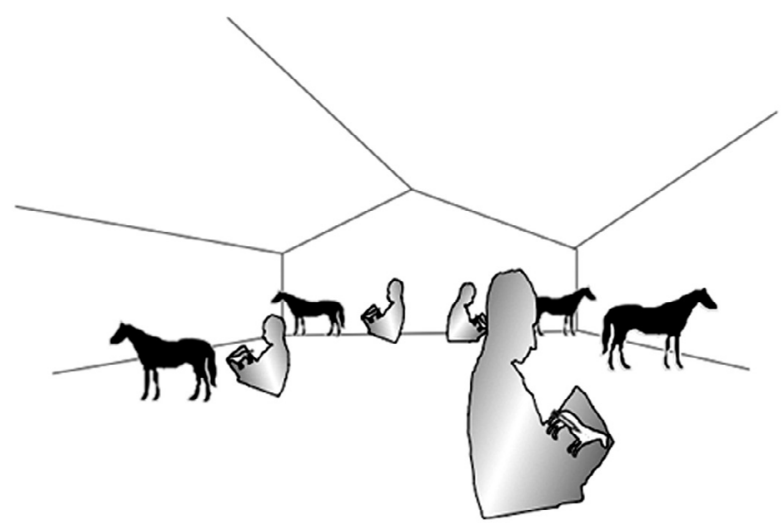

Figure 4. Design for counting mosquitoes for a total of 10 minutes ( 5 minutes on each side of the horse) each hour during the total six hours of observation. During this observation, the number of mosquitoes on face, neck, different parts of the body, and legs of each horse were also noted.)

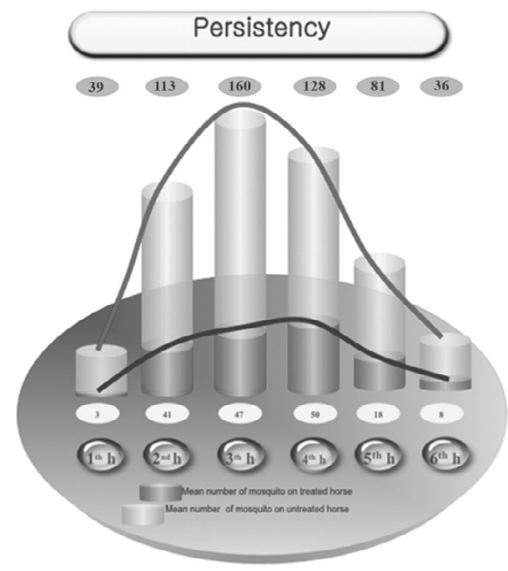

Figure 5. The graph above is the avarage of days, of mosquotoes hourly landed on UNTREATED Horse during 4 evenigs of testing ( all the mosquotoes landed during each hours and counted during 10 minutes) during 6 houres

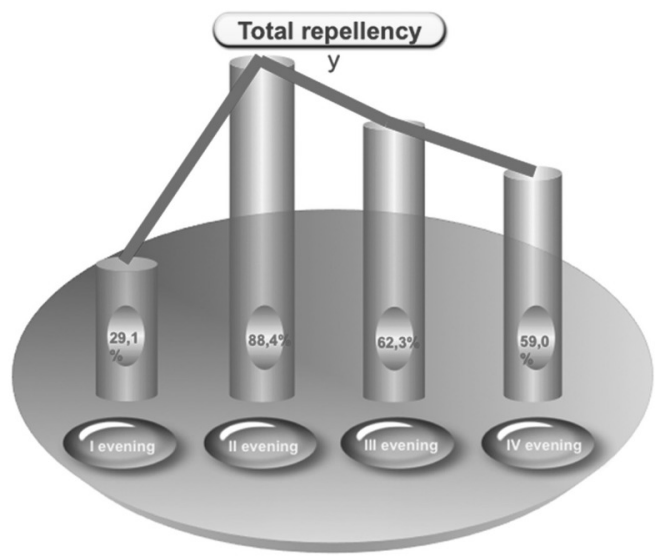

Figure 6. The persentage of repellency calculated as of \% less landed mosquotoes on Untreated horses during 4 evenings

effective and warrants further research concerning other nuisance and harmful insects in stable habitats and elsewhere.

Since, the product, here evaluated, is originally intended for topical use on persons [9] which has already demonstrated its effectiveness, and considering that the use of repellents is recommended in the protocols for the fight against cutaneous leishmanaisis, by using it against its vectors, therefore, the use of a completely botanical one, as Repel Care ${ }^{\varpi}$ is esteemed and also considered to be adopted as a local potent expedient approach. The effectiveness of this repellent will be accurately assessed (Figure 8 and 9).

Therefore, the performance study of this product will be pursued as of a novel product by technicians of a local Health Network of "Iran University of Medical Science" of Iran that are looking forward to receiving required authorization to starting the assessment of this product, and the next future inclusion in the protocols for control of

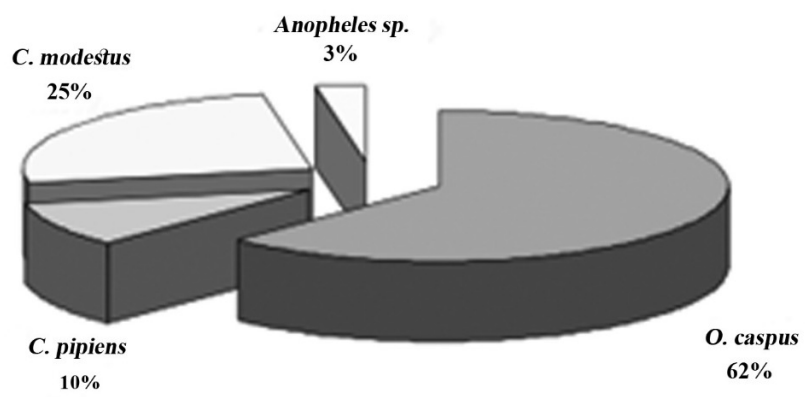

Figure 7. Qualitative and quantitative composition of adult mosquitotoes in CDC trapplaces East of the test shed

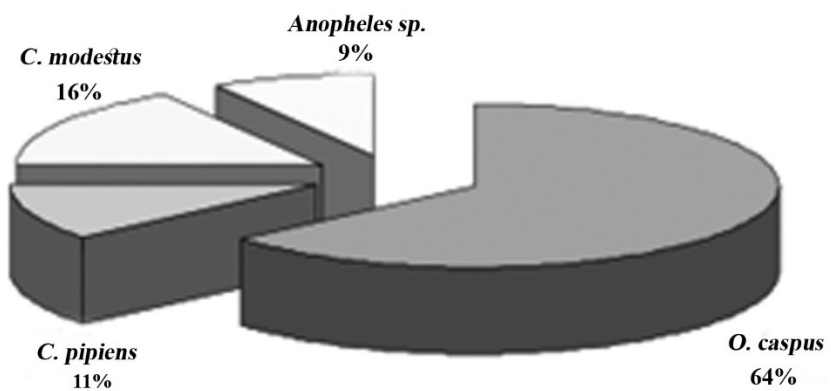

Figure 8. Qualitative and quantitative composition of adult mosquitoes in CDC trap placed North of the test shed

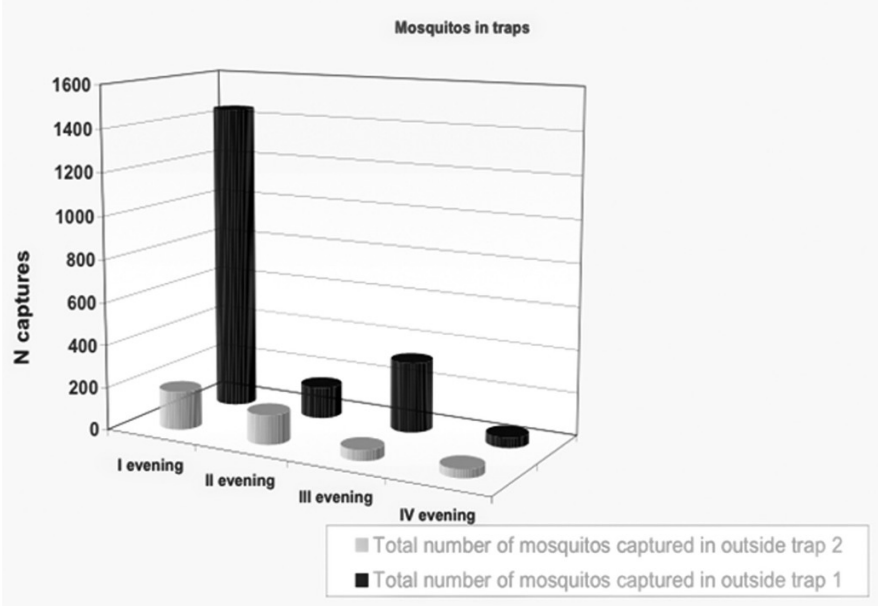

Figure 9. Simultaneous collection of total capture of mosquitoes each evening with 2 traps during obeservation of landed mosquitoes on horses (how many mosquitoes outside in traps meanwhile counting during of same interval on horses (we can call it The Pressure of mosquitoes outside as designe in Figure 2 
the sand fly, implementing the same method applied for the tests on the horses.

\section{Conclusion}

This preliminary evaluation of Repel Care revealed the effectiveness of this botanical mosquito repellent against horses. The owners of the horses were satisfied with the results although complete repellence was not achieved at the rates of the repellent used in this study. Because of the densely hairy bodies of this animal, higher rates of the repellent on horses may be required for better results. Considering the safety features associated with botanical repellents (compared to chemical adulticiding, or use of chemical repellents), further studies to fine tune the effective rates of this repellent on horses as well as standardization of repellent application method for its uniform distribution are suggested.

\section{Acknowledgment}

Sincere gratitude is expressed to CB QUARTER HORSE TRAINING SCHOOL of Barbara Cuniolo, Adriana Arobba and Claudia Pastore, the owners and trainers of horses: "CLASSICAL JUSTICE", "CODY BENITO BAR", "HOT AS I WANNA BE" and "LIL SMART PEPPY". The hard work of Fabio Fossati, and Moritz Bitzhenner during the course of this evaluation is duly acknowledged. R. Chanon, C. Nackanun, T. Apiwat and T. Usavadee are thanked for the invention and the production of the tested repellent product.

\section{References}

1. Talbalaghi A (2000) Mosquito control in southern Alexandria's province of Piedmont Proc. 13th European SOVE meeting. Page 79. Proceeding of the International Symposium on Mosquitoes in Ricefields, organized by EMCA and University of Eastern Piedmont.

2. Report of (2005) Ente Nazionale Risi of Italian Ministry of Agriulture. Dati statistici delle superfici coltivate a riso in Italia, Page 2.

3. Talbalaghi A, Mosvca A, BalboL, Huancahuari M (2002) Rice cultivation and mosquito development in Piedmont (Italy). Proceeding 2nd EMCA Workshop- Bologna: 28-29.

4. Tawatsin A Asavadachannukorn P, U Thavara (2006) Repellency of eseential oils extracted from plants in Thailand against four mosquito vectors (Diptera: Culicidae) and oviposition deterent effects against Aedes aegypti. Southeast Asian J Trop Med Public Health: 37

5. Barnard DR, Ulrich Bernier, Xue RD, Mustapha Debboun (2000) Standards Methods for Testing Mosquito Repellents. American Society for Testing Materials Method Page 103

6. Reisen WK (2004) University of California, Center for Vectorborne Diseases, Department of Pathology, Microbiology and Immunology, School of Veterinary Vector Biology, Ecology and Control: 127-137.

7. Debboun M, Frances SP, Strickman D (2007) Insect repellents-Principles, Methods and USES, CRC Press, Boca Raton.

8. Xue RD, Ali A, Day JF (2007) Commercially available insect repellents and criteria for their use, pp: 405-415.

9. Debboun M, Frances S, Strickman D (2007) Insect repellents: Principles, methods and use. Taylor and Francis.

Copyright: (C2018 Talbalaghi A. This is an open-access article distributed under the terms of the Creative Commons Attribution License, which permits unrestricted use, distribution, and reproduction in any medium, provided the original author and source are credited. 